University of Wollongong

Research Online

Australian Institute for Innovative Materials -

Papers

Australian Institute for Innovative Materials

$1-1-2016$

Collapse and reappearance of magnetic orderings in spin frustrated TbMnO3 induced by Fe substitution

\author{
Fang Hong \\ Center for High Pressure Science and Technology Advanced Research, University of Wollongong, \\ Lawrence Berkeley National Laboratory \\ Binbin Yue \\ Center for High Pressure Science and Technology Advanced Research, Lawrence Berkeley National \\ Laboratory \\ Jian Li Wang \\ University of Wollongong, Australian Nuclear Science And Technology Organisation, jianli@uow.edu.au \\ Andrew J. Studer \\ Australian Nuclear Science And Technology Organisation \\ Chunsheng Fang \\ University of Wollongong, cf904@uowmail.edu.au
}

See next page for additional authors

Follow this and additional works at: https://ro.uow.edu.au/aiimpapers

Part of the Engineering Commons, and the Physical Sciences and Mathematics Commons

Research Online is the open access institutional repository for the University of Wollongong. For further information contact the UOW Library: research-pubs@uow.edu.au 


\title{
Collapse and reappearance of magnetic orderings in spin frustrated TbMnO3 induced by Fe substitution
}

\author{
Abstract \\ We studied the temperature dependent magnetic phase evolution in spin frustrated $\mathrm{TbMnO}$ affected by

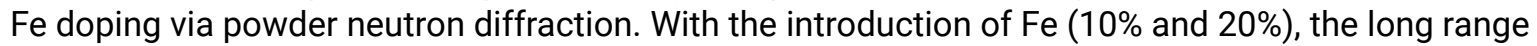 \\ incommensurate magnetic orderings collapse. When the Fe content is increased to $30 \%$, a long-range \\ antiferromagnetic ordering develops, while a spin reorientation transition is found near $35 \mathrm{~K}$ from a \\ canted G-type antiferromagnetic ordering to a collinear G-type antiferromagnetic ordering. This work \\ demonstrates the complex magnetic interactions existing in transition metal oxides, which helps to \\ understand the frustrated spin states in other similar systems and design magnetic materials as well. \\ Disciplines \\ Engineering | Physical Sciences and Mathematics

\section{Publication Details} \\ Hong, F., Yue, B., Wang, J. Li., Studer, A., Fang, C., Wang, X., Dou, S. \& Cheng, Z. (2016). Collapse and \\ reappearance of magnetic orderings in spin frustrated TbMnO3 induced by Fe substitution. Applied \\ Physics Letters, 109 (10), 102401-1-102401-5.

\section{Authors} \\ Fang Hong, Binbin Yue, Jian Li Wang, Andrew J. Studer, Chunsheng Fang, Xiaolin Wang, Shi Xue Dou, and \\ Zhenxiang Cheng
}




\title{
Collapse and reappearance of magnetic orderings in spin frustrated $\mathrm{TbMnO}_{3}$ induced by $\mathrm{Fe}$ substitution
}

\author{
Fang Hong, ${ }^{1,2,3}$ Binbin Yue,${ }^{2,3, a)}$ Jianli Wang, ${ }^{1,4}$ Andrew Studer, ${ }^{4}$ Chunsheng Fang, ${ }^{1}$ \\ Xiaolin Wang, ${ }^{1}$ Shixue Dou, ${ }^{1}$ and Zhenxiang Cheng ${ }^{1, a)}$ \\ ${ }^{1}$ Institute for Superconducting and Electronic Materials, Australian Institute of Innovative Materials, \\ University of Wollongong, Innovation Campus, Squires Way, North Wollongong, New South Wales 2500, \\ Australia \\ ${ }^{2}$ Center for High Pressure Science and Technology Advanced Research, 1690 Cailun Rd. Pudong, \\ Shanghai 201203, People's Republic of China \\ ${ }^{3}$ The Advanced Light Source, Lawrence Berkeley National Laboratory, 1 Cyclotron Rd, MS 80R0114, \\ Berkeley, California 94720, USA \\ ${ }^{4}$ The Australian Nuclear Science and Technology Organization (ANSTO), Bragg Institute, Lucas Heights, \\ New South Wales 2234, Australia
}

(Received 7 July 2016; accepted 28 August 2016; published online 7 September 2016)

\begin{abstract}
We studied the temperature dependent magnetic phase evolution in spin frustrated $\mathrm{TbMnO}_{3}$ affected by Fe doping via powder neutron diffraction. With the introduction of $\mathrm{Fe}(10 \%$ and $20 \%)$, the long range incommensurate magnetic orderings collapse. When the Fe content is increased to $30 \%$, a long-range antiferromagnetic ordering develops, while a spin reorientation transition is found near $35 \mathrm{~K}$ from a canted G-type antiferromagnetic ordering to a collinear G-type antiferromagnetic ordering. This work demonstrates the complex magnetic interactions existing in transition metal oxides, which helps to understand the frustrated spin states in other similar systems and design magnetic materials as well. Published by AIP Publishing.
\end{abstract}

[http://dx.doi.org/10.1063/1.4962465]

Frustrated spin systems have aroused great interests due to their complex magnetic interactions. ${ }^{1-7}$ In such kinds of frustrated magnetic systems, many unique magnetic structures and physical properties are observed. ${ }^{8-12}$ Magnetic monopoles have even been proposed to exist in some frustrated spin ice systems (for example, $\mathrm{Ho}_{2} \mathrm{Ti}_{2} \mathrm{O}_{7}$ and $\mathrm{Dy}_{2} \mathrm{Ti}_{2} \mathrm{O}_{7}$ ) ${ }^{13-16}$ Due to the competition in the anisotropic interactions among the magnetic ions at low temperature, a frustrated helical spin ordering can develop from the Mn sublattice in orthorhombic $\mathrm{TbMnO}_{3}$ and $\mathrm{DyMnO}_{3}$, and they can consequently give rise to ferroelectric ordering, which is why these compounds are called "multiferroic" materials. ${ }^{17-22}$ Because of the magnetic origin of ferroelectric ordering in these materials, and the broken spatial inversion and time-reversal symmetries, ${ }^{23}$ there is strong magnetoelectric coupling in these kinds of multiferroic materials, which is promising for more operations depending on more degrees of freedom and has benefits for the design of next-generation information storage systems. ${ }^{24-28}$

Meanwhile, the abundant phase transitions that occur in these frustrated multiferroic systems have inspired great interest as well. ${ }^{17,18,29-32}$ Orthorhombic $\mathrm{TbMnO}_{3}$ has a similar phase diagram to that of $\mathrm{DyMnO}_{3}{ }^{4}{ }^{4}$ Systematic neutron diffraction studies on $\mathrm{TbMnO}_{3}$ single crystal were conducted by Kajimoto et al. and Kenzelmann et al. ${ }^{33,34} \mathrm{TbMnO}_{3}$ experiences its antiferromagnetic (AFM) transition at $\sim 46 \mathrm{~K}$. When the temperature is between $46 \mathrm{~K}$ and $28 \mathrm{~K}, \mathrm{Mn}^{3+}$ spins are expected to first order along the $b$ axis (Pbnm space group) in an incommensurate magnetic (ICM) sinusoidal arrangement. Below $28 \mathrm{~K}$, an additional component of the $\mathrm{Mn}^{3+}$ magnetic

\footnotetext{
${ }^{\text {a) }}$ Authors to whom correspondence should be addressed. Electronic addresses: yuebb@hpstar.ac.cn and cheng@uow.edu.au
}

moment along the $c$ axis gives rise to a spiral magnetic ordering and breaks the inversion symmetry, leading to the observation of polarization $(P s)$ reflection along the $c$ axis. ${ }^{18,20}$ The rare earth $\mathrm{Tb}^{3+}$ ions enter into antiferromagnetic ordering around $7 \mathrm{~K} .^{33,34}$

Fe doping is an effective way to tune the magnetic interaction in manganese oxide because of the extra electron contribution from $\mathrm{Fe} 3 d$ orbital. The $\mathrm{Fe}$ doping effect on the frustrated magnetic states in $\mathrm{DyMnO}_{3}$ has been investigated by general magnetic measurements in the laboratory, and a similar phase scheme has been proposed by two different research groups. ${ }^{35,36}$ The real magnetic states in this kind of system are still full of uncertainty, however, since they are only based on general magnetic measurements. To reveal how the magnetic interaction evolves with Fe doping in such kinds of magnetic systems, we carried out temperature dependent powder neutron diffraction experiments on an alternative system $\mathrm{TbMnO}_{3}$, a promising multiferroic material for device application, in which the ferroelectric ordering can be enhanced by pressure and the ferroelectric domain wall can be well controlled by the external magnetic field without shape and position change. ${ }^{21,22}$ Interestingly, a few different types of unexpected magnetic behavior are observed in our neutron experiment. This work provides meaningful information on understanding the frustrated spin interactions in this kind of magnetic system.

Big volumes of samples were used to collect the neutron diffraction patterns from $6 \mathrm{~K}$ to $300-430 \mathrm{~K}$ and the experiment details are given in the supplementary material. Enlargements of the $20^{\circ}-50^{\circ}$ region, showing the magnetic contribution, are presented in Figure 1, and the full patterns are displayed in Figure S1 in the supplementary material. 

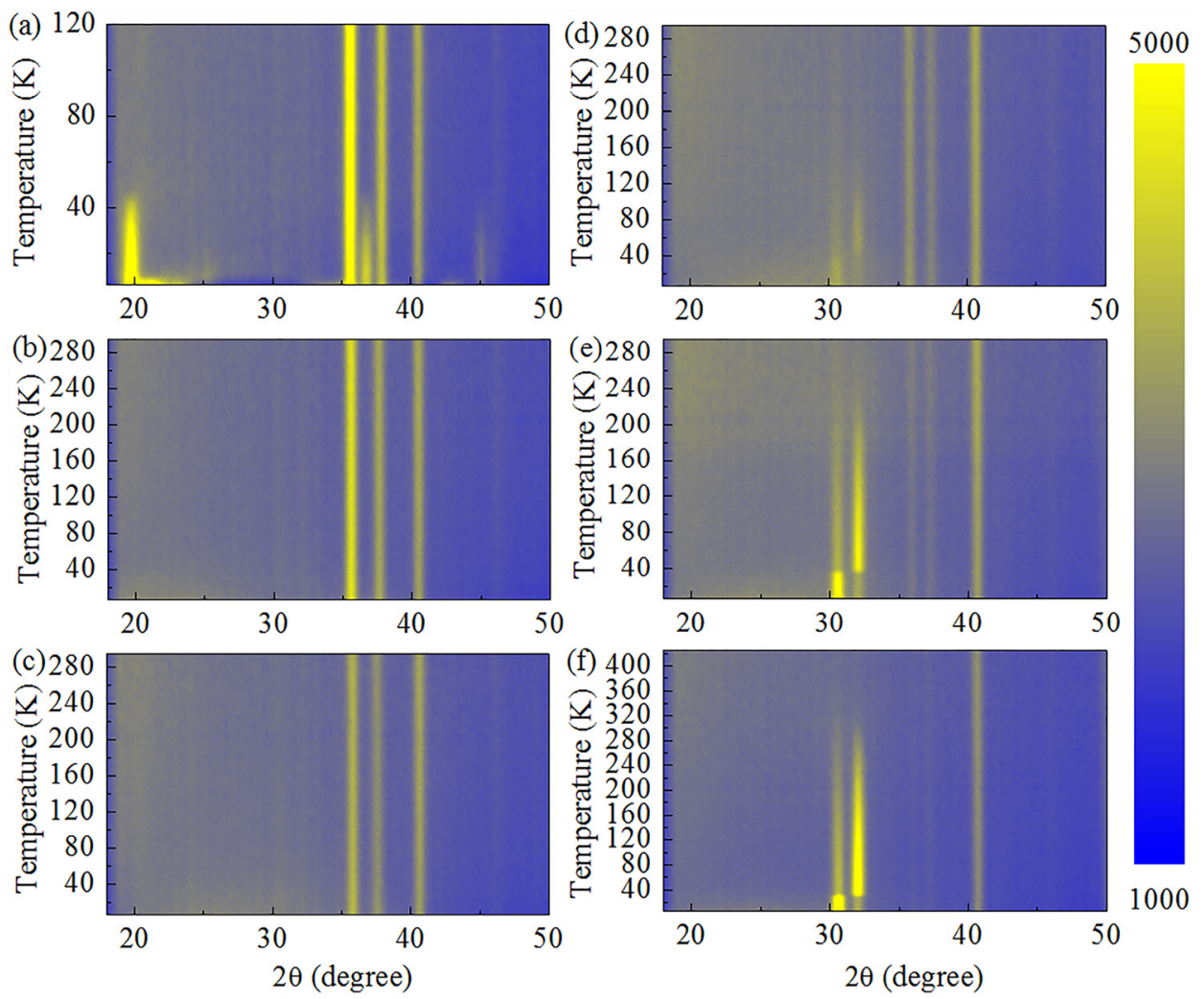

FIG. 1. Powder neutron diffraction patterns of $\mathrm{TbMn}_{1-x} \mathrm{Fe}_{x} \mathrm{O}_{3}$. (a)-(f) The temperature dependent neutron diffraction patterns for the samples with $x=0$, $0.1,0.2,0.3,0.4,0.5$, respectively.
Most peaks exhibited a nuclear shift toward higher diffraction angles due to the contraction of the unit cell at low temperature. There are also some peaks that are shifted toward lower angles, suggesting that at least one of the three lattice parameters becomes larger at low temperature. In the low diffraction angle region, ranging from $18^{\circ}$ to $35^{\circ}$, a clear extra peak can be observed near $20^{\circ}$ for pure $\mathrm{TbMnO}_{3}$, as shown in Figure 1(a), which is ascribed to the antiferromagnetic transition. Extra peaks can also be seen around $40^{\circ}$ with increasing intensity at a slightly lower temperature, suggesting the second antiferromagnetic transition. The refinement results confirm the pure orthorhombic phase for all samples with space group Pbnm.

When Fe is introduced into the $\mathrm{TbMnO}_{3}$, it is supposed to shift the antiferromagnetic transition to higher temperature, according to a previous related study on $\mathrm{DyMnO}_{3} .{ }^{35,36}$ On the contrary, we cannot see any magnetic contribution to the neutron diffraction patterns for samples with Fe content of $10 \%$ and $20 \%$. For the whole temperature range, we can only obtain the nuclear contribution, as shown in Figures 1(b), 1(c), S1(b), and S1(c). This is unexpected, and the current neutron results clearly demonstrate that the magnetic ordering collapses with $\mathrm{Fe}$ doping. The previously existing long-range incommensurate magnetic ordering in $\mathrm{TbMnO}_{3}$ is totally suppressed, and the system is likely to be in a highly frustrated spin state, which is almost identical to the paramagnetic state. In contrast to the pure paramagnetic state, there is remnant magnetic moment on the hysteresis magnetic loops for these two samples, indicating that there is a dynamically strong spin interaction, and this interaction is easily broken by the external magnetic field (see Figure S2 in the supplementary material). To find out whether there is a spin liquid state at low temperature in $\mathrm{TbMn}_{0.9} \mathrm{Fe}_{0.1} \mathrm{O}_{3}$ and
$\mathrm{TbMn}_{0.8} \mathrm{Fe}_{0.2} \mathrm{O}_{3}$, we looked at the history effect on the temperature dependent moment. Our zero-field-cooled (ZFC) and field-cooled (FC) curves (presented in Figure S3 in the supplementary material) show a clear breakaway below $15 \mathrm{~K}$ and $20 \mathrm{~K}$ in $\mathrm{TbMn}_{0.9} \mathrm{Fe}_{0.1} \mathrm{O}_{3}$ and $\mathrm{TbMn}_{0.8} \mathrm{Fe}_{0.2} \mathrm{O}_{3}$, respectively. The different behavior between the ZFC and FC processes excludes the existence of a spin liquid state and suggests the occurrence of a spin-glass-like state. ${ }^{37,38}$ The appearance of spin-glass-like state could be due to the competition interaction among $\mathrm{Mn}-\mathrm{O}-\mathrm{Mn}, \mathrm{Fe}-\mathrm{O}-\mathrm{Fe}, \mathrm{Mn}-\mathrm{O}-\mathrm{Fe}$, and even $\mathrm{Tb}-\mathrm{Mn}$ or $\mathrm{Tb}-\mathrm{Fe}{ }^{39}$ Unfortunately, current work cannot define the exact competition interaction. There is also another possibility, that the small amount of Fe causes random disorder in the exchange interaction and makes it hard to form long range ordering. A similar phenomenon has been observed in $\mathrm{Al}$-doped $\mathrm{DyMnO}_{3}$, in which more than $3 \% \mathrm{Al}$ will strongly suppress the electric polarization due to the breaking of the long range ordering of Mn and Dy. ${ }^{19}$

This highly frustrated spin state will be broken once the Fe ratio increases to $30 \%$, as shown in Figure 1(d). Two extra peaks appear in the low temperature range around $30^{\circ}$. Above $120 \mathrm{~K}$, there is only a nuclear contribution to the diffraction. Near $36 \mathrm{~K}$, there is a change in the relative intensities of these two peaks, indicating a spin reorientation transition. The different magnetic peak positions from pure $\mathrm{TbMnO}_{3}$ also suggest a totally different magnetic ordering in $\mathrm{TbMn}_{0.7} \mathrm{Fe}_{0.3} \mathrm{O}_{3}$. Similar magnetic behavior is found in the samples with Fe ratios of $40 \%$ and $50 \%$, as shown in Figures 1(e) and 1(f). The spin reorientation transition temperature shifts to lower temperature as the Fe content increases, while the antiferromagnetic-paramagnetic transition (Néel) temperature shifts to higher temperature. The spin reorientation transition has also been confirmed by magnetic measurements on 
a physical properties measurement system (PPMS) (see Figure S4 in the supplementary material). This magnetic behavior is to some extent similar to that in the $\mathrm{DyMn}_{1-x} \mathrm{Fe}_{x} \mathrm{O}_{3}$ system, in which the spin reorientation transition occurs when the Fe ratio is above $50 \%$. The presence of the spin reorientation transition further suggests that there is strong competition among the spin-exchange interactions and that frustrated spin states can be induced by Fe doping.

The trends in the evolution of the lattice parameters and unit cell volumes with temperature for all samples are shown in Figure 2. Several representative refinement results are given in Figure S5 and refinement information can be found in Tables S1-S3 and Figure S6 in the supplementary material. As mentioned above, the unit cell volume becomes smaller as the temperature cools down. In the case of the lattice parameters, both $b$ and $c$ continuously decrease as the temperature cools down, while $a$ decreases at first and then increases in the lower temperature range starting near $200 \mathrm{~K}$. There is no sharp change in the unit cell volume or lattice parameters, which suggests that the magnetic transitions in the current system are all second order transitions.

The magnetic information obtained from refinement results is displayed in Figure 3. For the pure $\mathrm{TbMnO}_{3}$, the magnetic moment contributed by the Mn sublattice along the $a$ axis increases as the temperature decreases, while the propagation vector $k_{y}$ first decreases down to $\sim 30 \mathrm{~K}$, below which the propagation vector shows almost no change, as can be seen in Figure 3(a). This is strongly consistent with previous neutron studies based on single crystal $\mathrm{TbMnO}_{3} .{ }^{33,34} \mathrm{As}$ shown by the neutron mapping results for samples with $\mathrm{Fe}$ content of $10 \%$ and $20 \%$, there are no magnetic contributions
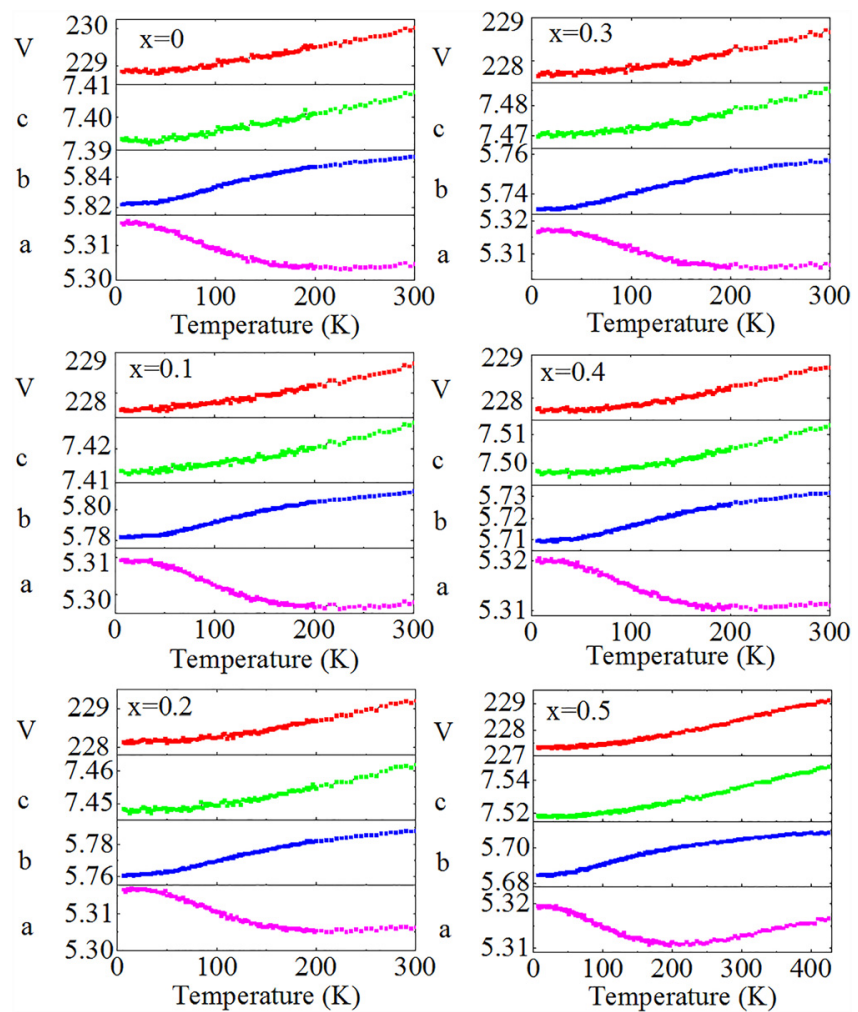

FIG. 2. Temperature dependence of the lattice parameters and volumes obtained from the refinement results (units: lattice parameters, $a, b, c$ : $\AA$; volume of unit cell, $\AA^{3}$ ). over the entire temperature range. For samples with Fe content of $30 \%, 40 \%$, and $50 \%$, the $\mathrm{Mn} / \mathrm{Fe}$ spins first order along the $b$ axis with a G-type antiferromagnetic configuration, with the moment increasing gradually as the sample is cooled down, and then the G-type antiferromagnetic $\mathrm{Mn} / \mathrm{Fe}$ spins start to rotate to the $a$ axis near $40 \mathrm{~K}$, as shown in Figures 3(b)-3(d). The moment increases sharply along the $a$ axis just after the phase transition and reaches a plateau. The average moment increases as the Fe content increases. Meanwhile, a ferromagnetic component appears along the $b$ axis. This behavior is quite different from that in $\mathrm{TbFeO}_{3}$, in which spin reorientation is only found near $6.5 \mathrm{~K}$, and the strong interaction between $\mathrm{Tb}-\mathrm{Fe}$ is responsible for this transition. ${ }^{39}$ The spin reorientation here is somewhat similar to that in $\mathrm{DyFeO}_{3}$, in which there is a spin reorientation near $35 \mathrm{~K}$, but the spin reorientation process here is totally opposite to that of $\mathrm{DyFeO}_{3} \cdot{ }^{40}$ Apparently, the origin of the spin reorientation in the current system is mainly from the competitive interaction among $\mathrm{Fe}$ and $\mathrm{Mn}$ ions. The real stoichiometry of $\mathrm{Mn}$ and $\mathrm{Fe}$ content is also obtained and the results are: $\mathrm{TbMn}_{0.9} \mathrm{Fe}_{0.1} \mathrm{O}_{3}$ $\left(\mathrm{TbMn}_{0.927} \mathrm{Fe}_{0.073} \mathrm{O}_{3}\right) ; \mathrm{TbMn}_{0.8} \mathrm{Fe}_{0.2} \mathrm{O}_{3}\left(\mathrm{TbMn}_{0.766} \mathrm{Fe}_{0.234} \mathrm{O}_{3}\right)$; $\mathrm{TbMn}_{0.7} \mathrm{Fe}_{0.3} \mathrm{O}_{3} \quad\left(\mathrm{TbMn}_{0.715} \mathrm{Fe}_{0.285} \mathrm{O}_{3}\right) ; \quad \mathrm{TbMn}_{0.6} \mathrm{Fe}_{0.4} \mathrm{O}_{3}$ $\left(\mathrm{TbMn}_{0.583} \mathrm{Fe}_{0.417} \mathrm{O}_{3}\right) ; \mathrm{TbMn}_{0.5} \mathrm{Fe}_{0.5} \mathrm{O}_{3}\left(\mathrm{TbMn}_{0.432} \mathrm{Fe}_{0.568} \mathrm{O}_{3}\right)$.

Based on the above discussion, we can briefly summarize the $\mathrm{Fe}$ doping effect on the magnetic structure in $\mathrm{TbMnO}_{3}$. The long-range ordering of the incommensurate spin state in pure $\mathrm{TbMnO}_{3}$ can be destroyed by a small amount of $\mathrm{Fe}$, which drives the system to a highly frustrated spin state (spin-glass-like state). Increasing the level of $\mathrm{Fe}$ doping helps to develop long-range orderings, which are completely different from those in pure $\mathrm{TbMnO}_{3}$. Due to the complex exchange interaction among $\mathrm{Mn}-\mathrm{Mn}, \mathrm{Fe}-\mathrm{Fe}, \mathrm{Mn}-$ $\mathrm{Fe}$, and even $\mathrm{Tb}-\mathrm{Fe}$ or $\mathrm{Tb}-\mathrm{Mn}$, a spin reorientation transition is found when the $\mathrm{Fe}$ ratio is increased to $30 \%$. The spin reorientation transition temperature does not shift too much when the Fe ratio increases from $30 \%$ to $50 \%$, while the Néel temperature increases quickly with the Fe content. The spin reorientation transition follows a transition sequence from a G-type antiferromagnetic ordering along the $b$ axis $(G y)$ to a canted G-type antiferromagnetic ordering $(G x F y)$. A proposed magnetic phase diagram has been plotted in Figure 4(a) and the magnetic structures for Gy and GxFy have been given in Figures 4(b) and 4(c), respectively. To avoid misunderstanding on this diagram, the known transition temperatures are only those observed in $\mathrm{TbMnO}_{3}$ and Fe-doped samples with $x=0.3,0.4$, and 0.5 , based on current experiment. Beyond those, all boundary data are speculated.

In spin frustrated $\mathrm{Mn}_{1-\mathrm{x}} \mathrm{Co}_{\mathrm{x}} \mathrm{WO}_{4}$ system, the Co doping is found to help to stabilize the multiferroic ordering and plays a role as same as external magnetic field. ${ }^{41}$ The magnetic ordering changes from a commensurate ordering to an incommensurate ordering. Actually, this is a transition from a non-frustrated spin state to frustrated spin state. With higher doping rate, there is a spin flop process, which is also regarded as a spin reorientation transition due to the competition of spin exchange interactions. With the introduction of non-magnetic Mo into $\mathrm{MnWO}_{4}$, the magnetic structure does not change but the transition temperature increases with Mo substitution. ${ }^{6}$ There is also a detailed report on the transition metal doped $\mathrm{FeVO}_{4}$, in which the magnetic ordering does 

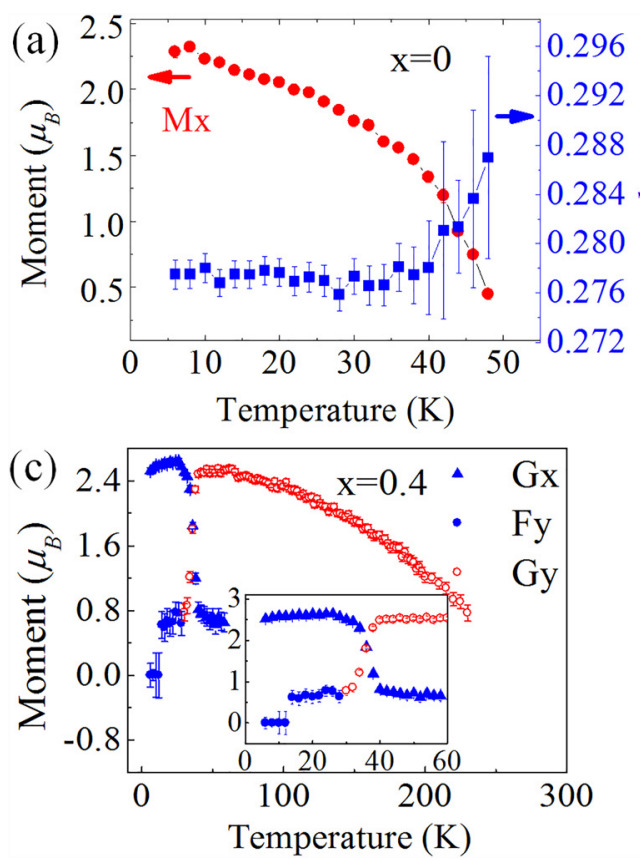
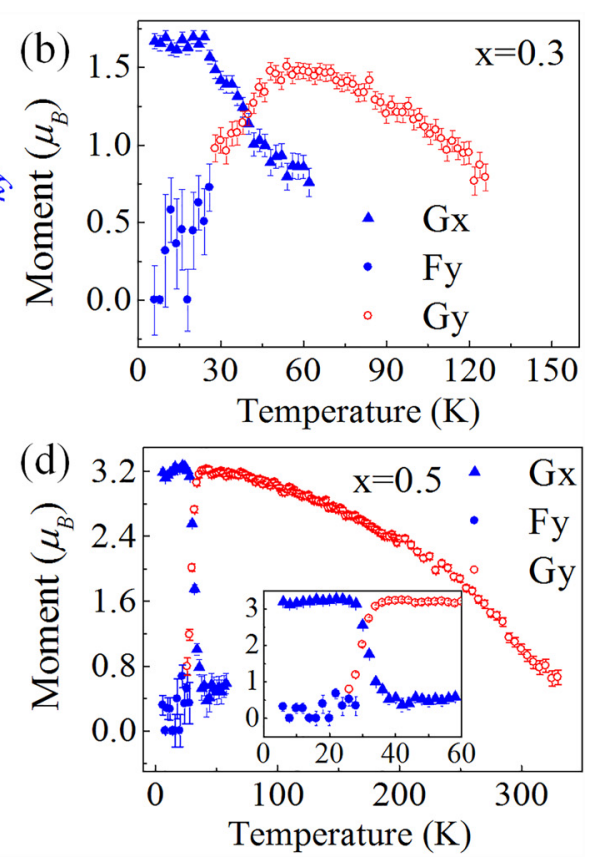

FIG. 3. Magnetic information based on refinement. (a) Temperature dependence of the magnetic moment along the $a$ axis with propagation vector along the $b$ axis for pure $\mathrm{TbMnO}_{3}$. (b)-(d) Temperature dependence of the magnetic moments for samples with $x=0.3,0.4$, and 0.5 , respectively. The insets show enlargements of the respective low temperature regions. not change no matter it is magnetic doping nor nonmagnetic doping, and the ferroelectric ordering is even kept at a big doping rate. ${ }^{42}$ The nonmagnetic doping on frustrated $\mathrm{Ni}_{3} \mathrm{~V}_{2} \mathrm{O}_{8}$ only plays a dilution effect on the magnetic structure. ${ }^{43}$ The magnetic $\mathrm{Cu}$ doping on $\mathrm{Ni}_{3} \mathrm{~V}_{2} \mathrm{O}_{8}$, however, can suppress the frustrated magnetic ordering and help to develop a possible non-frustrated ferromagnetic ordering when the doping rate is higher than $0.1{ }^{44}$ The magnetic doping effect is to some extent dependent on the types of magnetic elements. $\mathrm{Co}$ doping in $\mathrm{Ni}_{3} \mathrm{~V}_{2} \mathrm{O}_{8}$ does not break the frustrated multiferroic ordering even if the doping is higher than 0.3. Different from the frustrated spin systems mentioned above, either the spiral or collinear magnetic ordering, expected existing in magnetic $\mathrm{Mn} / \mathrm{Fe}$ sublattice, is totally absent in current $\mathrm{TbMn}_{1-\mathrm{x}} \mathrm{Fe}_{\mathrm{x}} \mathrm{O}_{3}$ system at low doping rate. Even compared with the $\mathrm{DyMn}_{1-\mathrm{x}} \mathrm{Fe}_{\mathrm{x}} \mathrm{O}_{3}$ system, in which there is no spin reorientation transition and ferromagnetic ordering at the same doping rate, the magnetic interaction in $\mathrm{TbMn}_{1-\mathrm{x}} \mathrm{Fe}_{\mathrm{x}} \mathrm{O}_{3}$ is much complicated. It seems hard to get a completely consistent trend for the doping effect on the

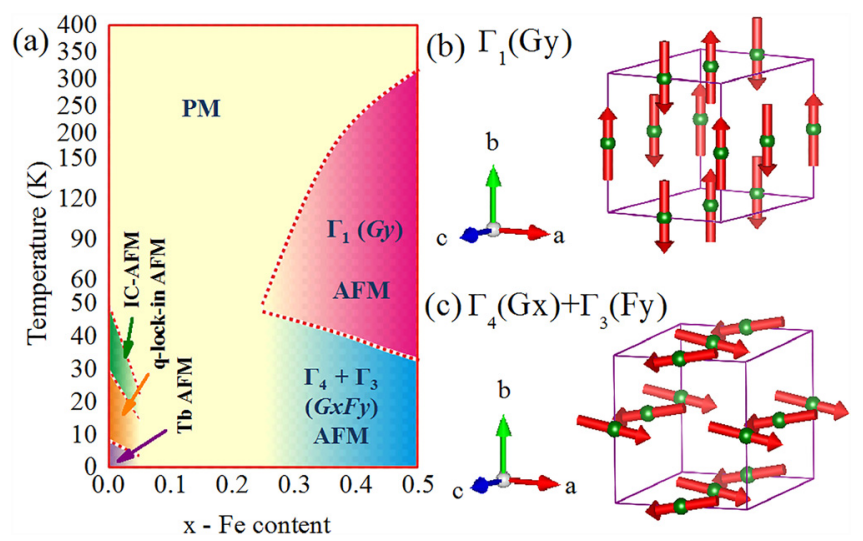

FIG. 4. Proposed magnetic phase diagram and representative magnetic structures of $\mathrm{TbMn}_{1-x} \mathrm{Fe}_{x} \mathrm{O}_{3}$ system based on neutron results. (a) Proposed phase diagram. (b) and (c) The magnetic structures for $\Gamma_{1}(G y)$ and $\Gamma_{4}+\Gamma_{3}$ $(G x F y)$, respectively. magnetic structures in different frustrated spin systems. Just because of such complexity and versatility, there are more rooms to tune the magnetic ordering and other ordering, which can help to design more magnetic materials and extend potential spintronic device application. Meanwhile, this work will help to understand the similar magnetic behavior in other systems.

In summary, the structural evolution and magnetic behavior of $\mathrm{TbMnO}_{3}$ with $\mathrm{Fe}$ doping has been investigated systemically by powder neutron diffraction. For all the samples, both the $b$ and the $c$ axis become shorter when the temperature is cooled down, while the $a$ axis contracts at first and then expands below $200 \mathrm{~K}$. All the unit cell volumes shrink as the temperature decreases. In $\mathrm{TbMnO}_{3}$, incommensurate spin orderings are observed, and our results are consistent with a previous single crystal study. The introduction of small amounts of Fe completely destroys the long-range spin ordering and pushes the systems $\left(\mathrm{TbMn}_{0.9} \mathrm{Fe}_{0.1} \mathrm{O}_{3}\right.$ and $\left.\mathrm{TbMn}_{0.8} \mathrm{Fe}_{0.2} \mathrm{O}_{3}\right)$ to a highly frustrated spin state. With further increases in Fe content (30\%-50\%), a spin reorientation process is observed near $35 \mathrm{~K}$, and this process follows a transition sequence from a collinear G-type antiferromagnetic ordering along the $b$ axis $(G y)$ to a canted G-type antiferromagnetic ordering $(G x F y)$. This work will provide a deeper understanding of the frustrated spin state and the complex magnetic interactions in similar systems.

See supplementary material for the full neutron diffraction patterns, magnetic hysteresis loops of $\mathrm{TbMn}_{0.9} \mathrm{Fe}_{0.1} \mathrm{O}_{3}$ and $\mathrm{TbMn}_{0.8} \mathrm{Fe}_{0.2} \mathrm{O}_{3}$ at selected temperatures, ZFC-FC curves for samples with $\mathrm{Fe}$ ratios of $10 \%$ and $20 \%$, ZFC-FC curves for samples with Fe ratios of $30 \%, 40 \%$, and $50 \%$, representative refinement results, and data analysis details.

Z. X. Cheng thanks the Australian Research Council through a Future Fellowship (FT 0990287). We thank Dr. Tania Silver for polishing the English. 
${ }^{1}$ W. Eerenstein, N. D. Mathur, and J. F. Scott, Nature 442, 759-765 (2006). ${ }^{2}$ S. T. Bramwell and M. J. P. Gingras, Science 294, 1495-1501 (2001).

${ }^{3}$ Y. Taguchi, Y. Oohara, H. Yoshizawa, N. Nagaosa, and Y. Tokura, Science 291, 2573-2576 (2001).

${ }^{4}$ L. Balents, Nature 464, 199-208 (2010).

${ }^{5}$ A. B. Cairns, M. J. Cliffe, J. A. M. Paddison, D. Daisenberger, M. G. Tucker, F.-X. Coudert, and A. L. Goodwin, Nat. Chem. 8, 442-447 (2016).

${ }^{6}$ L. Meddar, M. Josse, M. Maglione, A. Guiet, C. La, P. Deniard, R. Decourt, C. Lee, C. Tian, S. Jobic, M.-H. Whangbo, and C. Payen, Chem. Mater. 24, 353-360 (2012).

${ }^{7}$ L. Ortega-San-Martin, A. J. Williams, A. Storer, and J. P. Attfield, Chem. Mater. 21, 2436-2441 (2009).

${ }^{8}$ Y. K. Jeong, J.-H. Lee, S.-J. Ahn, S.-W. Song, H. M. Jang, H. Choi, and J. F. Scott, J. Am. Chem. Soc. 134, 1450-1453 (2012).

${ }^{9}$ F. D. Romero, L. Coyle, and M. A. Hayward, J. Am. Chem. Soc. 134, 15946-15952 (2012).

${ }^{10}$ L. Clark and P. Lightfoot, Nat. Chem. 8, 402-404 (2016).

${ }^{11}$ N. E. Amuneke, J. Tapp, C. R. de la Cruz, and A. Möller, Chem. Mater. 26, 5930-5935 (2014).

${ }^{12}$ H. Luo, J. W. Krizan, E. M. Seibel, W. Xie, G. S. Sahasrabudhe, S. L. Bergman, B. F. Phelan, J. Tao, Z. Wang, J. Zhang, and R. J. Cava, Chem. Mater. 27, 6810-6817 (2015).

${ }^{13}$ C. Castelnovo, R. Moessner, and S. L. Sondhi, Nature 451, 42-45 (2008).

${ }^{14}$ A. P. Ramirez, A. Hayashi, R. J. Cava, R. Siddharthan, and B. S. Shastry, Nature 399, 333-335 (1999).

${ }^{15}$ M. J. Harris, S. T. Bramwell, D. F. McMorrow, T. Zeiske, and K. W. Godfrey, Phys. Rev. Lett. 79, 2554-2557 (1997).

${ }^{16}$ Y. Tokiwa, T. Yamashita, M. Udagawa, S. Kittaka, T. Sakakibara, D. Terazawa, Y. Shimoyama, T. Terashima, Y. Yasui, T. Shibauchi, and Y. Matsuda, Nat. Commun. 7, 10807 (2016)

${ }^{17}$ J. Hemberger, F. Schrettle, A. Pimenov, P. Lunkenheimer, V. Y. Ivanov, A. A. Mukhin, A. M. Balbashov, and A. Loidl, Phys. Rev. B 75, 035118 (2007).

${ }^{18}$ T. Kimura, G. Lawes, T. Goto, Y. Tokura, and A. P. Ramirez, Phys. Rev. B 71, 224425 (2005).

${ }^{19}$ N. Zhang, K. F. Wang, S. J. Luo, T. Wei, X. W. Dong, S. Z. Li, J. G. Wan, and J.-M. Liu, Appl. Phys. Lett. 96, 252902 (2010).

${ }^{20}$ T. Kimura, S. Ishihara, H. Shintani, T. Arima, K. T. Takahashi, K. Ishizaka, and Y. Tokura, Phys. Rev. B 68, 060403 (2003).

${ }^{21}$ T. Aoyama, K. Yamauchi, A. Iyama, S. Picozzi, K. Shimizu, and T. Kimura, Nat. Commun. 5, 4927 (2014).

${ }^{22}$ M. Matsubara, S. Manz, M. Mochizuki, T. Kubacka, A. Iyama, N. Aliouane, T. Kimura, S. L. Johnson, D. Meier, and M. Fiebig, Science 348, 1112-1115 (2015).
${ }^{23}$ M. Mostovoy, Phys. Rev. Lett. 96, 067601 (2006)

${ }^{24}$ G. Catalan and J. F. Scott, Adv. Mater. 21, 2463-2485 (2009).

${ }^{25}$ C. A. F. Vaz, J. Hoffman, C. H. Anh, and R. Ramesh, Adv. Mater. 22, 2900-2918 (2010).

${ }^{26}$ I. Fina, L. Fabrega, X. Marti, F. Sanchez, and J. Fontcuberta, Appl. Phys. Lett. 97, 232905 (2010).

${ }^{27}$ V. Garcia, M. Bibes, L. Bocher, S. Valencia, F. Kronast, A. Crassous, X. Moya, S. Enouz-Vedrenne, A. Gloter, D. Imhoff, C. Deranlot, N. D. Mathur, S. Fusil, K. Bouzehouane, and A. Barthelemy, Science 327, 1106-1110 (2010)

${ }^{28}$ T. Lottermoser, T. Lonkai, U. Amann, D. Hohlwein, J. Ihringer, and M. Fiebig, Nature 430, 541-544 (2004).

${ }^{29}$ S. J. Luo, S. Z. Li, N. Zhang, T. Wei, X. W. Dong, K. F. Wang, and J. M. Liu, Thin Solid Films 519, 240-243 (2010).

${ }^{30}$ H. M. Jang, J. H. Park, S. W. Ryu, and S. R. Shannigrahi, Appl. Phys. Lett. 93, 252904 (2008).

${ }^{31}$ V. B. Naik and R. Mahendiran, J. Appl. Phys. 106, 123910 (2009).

${ }^{32}$ R. Muralidharan, T. H. Jang, C. H. Yang, Y. H. Jeong, and T. Y. Koo, Appl. Phys. Lett. 90, 012506 (2007).

${ }^{33}$ R. Kajimoto, H. Yoshizawa, H. Shintani, T. Kimura, and Y. Tokura, Phys. Rev. B 70, 012401 (2004).

${ }^{34}$ M. Kenzelmann, A. B. Harris, S. Jonas, C. Broholm, J. Schefer, S. B. Kim, C. L. Zhang, S. W. Cheong, O. P. Vajk, and J. W. Lynn, Phys. Rev. Lett. 95, 087206 (2005).

${ }^{35}$ F. Hong, Z. Cheng, H. Zhao, H. Kimura, and X. Wang, Appl. Phys. Lett. 99, 092502 (2011).

${ }^{36}$ F. K. Chiang, M. W. Chu, F. C. Chou, H. T. Jeng, H. S. Sheu, F. R. Chen, and C. H. Chen, Phys. Rev. B 83, 245105 (2011).

${ }^{37}$ M. J. P. Gingras, C. V. Stager, N. P. Raju, B. D. Gaulin, and J. E. Greedan, Phys. Rev. Lett. 78, 947-950 (1997).

${ }^{38}$ J. S. Gardner, S. R. Dunsiger, B. D. Gaulin, M. J. P. Gingras, J. E. Greedan, R. F. Kiefl, M. D. Lumsden, W. A. MacFarlane, N. P. Raju, J. E. Sonier, I. Swainson, and Z. Tun, Phys. Rev. Lett. 82, 1012-1015 (1999).

${ }^{39}$ O. Nikolov, I. Hall, S. N. Barilo, and S. A. Guretskii, J. Phys.: Condens. Matter 6, 3793 (1994).

${ }^{40}$ L. A. Prelorendjo, C. E. Johnson, M. F. Thomas, and B. M. Wanklyn, J. Phys. C: Solid State Phys. 13, 2567 (1980).

${ }^{41}$ Y. S. Song, J. H. Chung, J. M. S. Park, and Y. N. Choi, Phys. Rev. B 79, 224415 (2009).

${ }^{42}$ A. Kumarasiri, E. Abdelhamid, A. Dixit, and G. Lawes, Phys. Rev. B 91, 014420 (2015).

${ }^{43}$ P. Kharel, A. Kumarasiri, A. Dixit, N. Rogado, R. J. Cava, and G. Lawes, Philos. Mag. 89, 1923-1932 (2009).

${ }^{44}$ A. Kumarasiri and G. Lawes, Phys. Rev. B 84, 064447 (2011). 